\title{
Preparation of $\alpha$-Bromoketones and Thiazoles from Ketones with NBS and Thioamides in Ionic Liquids
}

\author{
Yuhta Izumisawa, Hideo Togo ${ }^{*}$ \\ Graduate School of Science, Chiba University, Chiba, Japan \\ E-mail: togo@faculty.chiba-u.jp \\ Received June 27, 2011; revised July 29, 2011; accepted August 5, 2011
}

\begin{abstract}
Ketones smoothly reacted with NBS in the presence of a catalytic amount of $p$-toluenesulfonic acid to give $\alpha$-bromoketones in good yields in typical ionic liquids, such as [bmim] $\mathrm{PF}_{6}$ and [bmpy] $\mathrm{Tf}_{2} \mathrm{~N}$, and the ionic liquids could be repeatedly used for the same reaction after the extraction of the $\alpha$-bromoketones. Then, the one-pot conversion of ketones into thiazoles by the treatment with NBS, and subsequently with thioamides could be also carried out in [bmim] $\mathrm{PF}_{6}$ and [bmpy] $\mathrm{Tf}_{2} \mathrm{~N}$, respectively Thus, [bmim] $\mathrm{PF}_{6}$ and $[\mathrm{bmpy}] \mathrm{Tf}_{2} \mathrm{~N}$ could be used as recyclable reaction media for the preparation $\alpha$-bromoketones and thiazoles from ketones.
\end{abstract}

Keywords: Ketone, $\alpha$-Bromoketone, Thiazole, NBS, Thioamide, Ionic Liquid

\section{Introduction}

Thiazoles are one of the most important heterocycles and known for their broad spectrum of biological activities $[1,2]$. Many natural and synthetic molecules containing the thiazole moiety play a significant role in the pharmaceutical industry due to their anti-inflammatory $[3,4]$, anti-HIV [5], anti-bacterial [6], anti-cancer [7] properties. Today, there are many methods for the preparation of the thiazole moiety [8-12]. One of the most excellent and efficient method is the Hantzsch thiazole synthesis $[13,14]$ that employs the reaction of $\alpha$-haloketones or $\alpha$-tosyloxyketones with thioamides. For the preparation of $\alpha$-bromonoketones from ketones, NBS ( $N$-bromosuccinimide) is well used [15], whereas HTIB [(hydroxy) (tosyloxy)iodobenzene] is the sole reagent for the direct preparation of $\alpha$-tosyloxyketones from ketones [16-22].

On the other hand, ionic liquids have grown in popularity as organic reaction media due to the promotion of ionic reactions and in view of environmental safety [2330]. Ionic liquids offer interesting and useful features that are advantageous to organic reactions such as negligible vapor pressure, nonflammability, high thermal stability, and easy reusability. In this regard, ionic liquids have been successfully used in the Friedel-Crafts reaction [31-33], hydrogenation [34-36], Diels-Alder reactions [37-39], Mizoroki-Heck, Suzuki-Miyaura, Sonogashira, and olefin metathesis reactions [40-44], Michael additions [45], oxidation [46-54], condensation reaction [55-59], formation of imines [60], 1,2-rearrangement
[61], esterification of carboxylic acids and carboxylates [62-65], Williamson ether synthesis [66-72], and the Grignard reaction [73,74]. We have reported efficient methods for the esterification of carboxylic acids and phosphonic acids with trialkyl orthoacetate in ionic liquid [75], the demethylation of $N, N$-dimethylanilines with phenyl chloroformate in ionic liquids [76], and the 3-exo -tet cyclization of 2,2-disubstituted 1,3-dihalopropanes with indium in ionic liquid [77], The $\alpha$-bromination of $\beta$-dicarbonyls and cyclic ketones with NBS in ionic liquids [78], and the aromatic ring bromination with NBS in ionic liquids $[79,80]$ have been reported as well. However, to the best of our knowledge, there are no synthetic studies that deal with the preparation of thiazoles from ketones with NBS and thioamides in ionic liquids. Here, as a part of our synthetic study of ionic liquids, we would like to report the preparation of $\alpha$-bromoketones and thiazoles from ketones, with NBS and thioamides in typical room-temperature ionic liquids.

\section{Results and Discussion}

The $\alpha$-bromination of ketones with NBS in the presence of a catalytic amount of $p$-toluenesulfonic acid ( $p$-TsOH) was carried out at room temperature in both chloroform and typical room-temperature ionic liquids, such as 1butyl-3-methylimidazolium hexafluorophosphate ([bmim] $\mathrm{PF}_{6}$ ), $N$-butyl- $N$-methylpyrrolidinium bis(trifluoromethanesulfonyl)imidate ([bmpy]Tf $\left.{ }_{2} \mathrm{~N}\right)$, and 1-butyl-3-methylimidazolium tetrafluoroborate $\left([\mathrm{bmim}] \mathrm{BF}_{4}\right)$, as shown 
in Table 1. As a result, the corresponding $\alpha$-bromoketones were obtained in good to high yields in chloroform, [bmim] $\mathrm{PF}_{6}$, and [bmpy] $\mathrm{Tf}_{2} \mathrm{~N}$, respectively. In contrast, the $\alpha$-bromination of ketones did not proceed at all in [bmim] $\mathrm{BF}_{4}$. It is probable that the proton derived from $p$-TsOH could not promote the formation of enol forms of ketones due to the interaction between the proton of $p$-TsOH and $\mathrm{BF}_{4}^{-}$. Practically, chemical shift of a hydrogen atom at 2-position of $\left[\mathrm{bmim}^{-B} \mathrm{BF}_{4}\right.$ is $9.42 \mathrm{ppm}$ $\left(\mathrm{CDCl}_{3}, \mathrm{TMS}\right)$, and is lower field than that of [bmim] $\mathrm{PF}_{6}$ (8.42 ppm, $\mathrm{CDCl}_{3}, \mathrm{TMS}$ ). This suggests that $\mathrm{BF}_{4}^{-}$in [bmim $\mathrm{BF}_{4}$ interacts strongly with a proton of $p$-TsOH. Moreover, the yields of $\alpha$-bromoketones in [bmim] $\mathrm{PF}_{6}$ and [bmpy] $\mathrm{Tf}_{2} \mathrm{~N}$ are higher overall than those in chloroform, especially when propiophenone and nonanophe-

Table 1. Conversion Ketones into $\alpha$-Bromoketones in ILs or $\mathrm{CHCl}_{3}$.

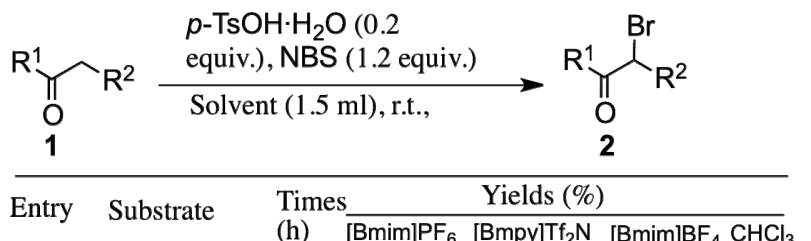

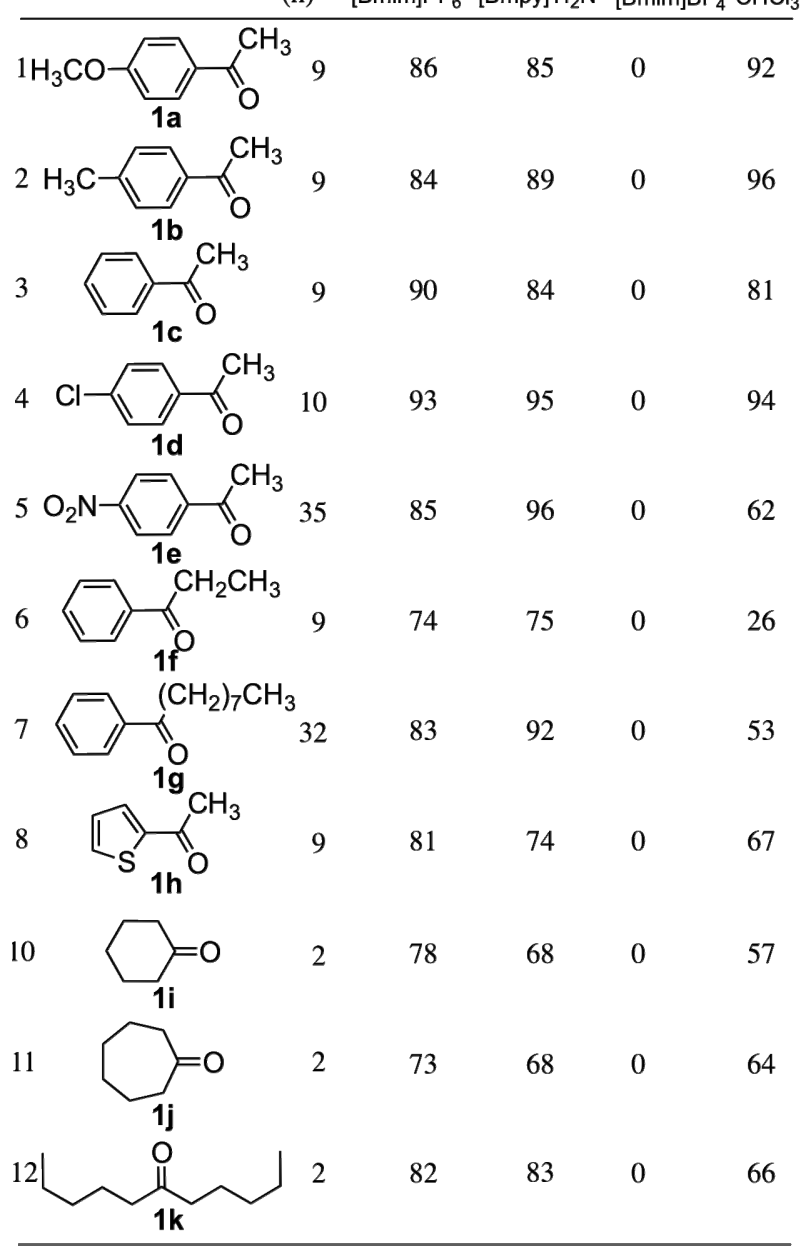

none were used as substrate (entries 6, 7). When an ionic liquid such as [bmim] $\mathrm{PF}_{6}$ was used, $\alpha$-bromoketone was obtained in good yields with good purity $(>80 \%)$ by simple ether extraction of the reaction mixture and the ionic liquid reaction medium could be reused for the same reaction up to the $7^{\text {th }}$ time while maintaining the high yields of $\alpha$-bromoketone, as shown in Table 2.

Then, the one-pot conversion of ketones to thiazoles in both chloroform and ionic liquids, such as [bmim] $\mathrm{PF}_{6}$ and [bmpy] $\mathrm{Tf}_{2} \mathrm{~N}$, was studied, as shown in Table 3. After the $\alpha$-bromination of ketones with NBS, thioamide and potassium carbonate were added to the reaction mixture, and the obtained mixture was stirred at room temperature. Overall, the yields in [bmim] $\mathrm{PF}_{6}$ and [bmpy] $\mathrm{Tf}_{2} \mathrm{~N}$ were higher than those in chloroform, particularly, when propiophenone with thiobenzamide, acetophenone with $p$-methoxythiobenzamide, and acetophenone with thioacetamide were used (entries $6,10,12$ ). In the reaction with acetophenone in [bmim] $\mathrm{PF}_{6}$, thiazoles were obtained in good yields with moderate purity $(>70 \%)$ by ether extraction, and the ionic liquid reaction medium could be reused for the same reaction, maintaining the good yields of thiazole up to the $5^{\text {th }}$ time, as shown in Table 4.

\section{Conclusions}

Typical room temperature ionic liquids, such as [bmim] $\mathrm{PF}_{6}$ and [bmpy] $\mathrm{Tf}_{2} \mathrm{~N}$ could be used for the conversion of ketones to $\alpha$-bromoketones with NBS and the conversion of ketones to thiazoles with NBS and subsequently thioamides in a one-pot manner. $\alpha$-Bromoketones and thiazoles could be obtained in good yields with good purity by simple ether extraction, and the ionic liquid reaction media could be reused for the same reaction while maintaining good yields and purity of the products. The present method offers a green approach to the preparation of $\alpha$-bromoketones and thiazoles in good yields with good purity from ketones with NBS and subsequently thioamides at room temperature.

\section{Experimental Section}

\subsection{General}

${ }^{1} \mathrm{H}$ NMR and ${ }^{13} \mathrm{C}$ NMR spectra were obtained on JEOL-

Table 2. Recyclic Use for conversion of acetophenpne to $\alpha$ Bromoketones from acetophenone in $[\mathrm{Bmim}] \mathrm{PF}_{6}$.

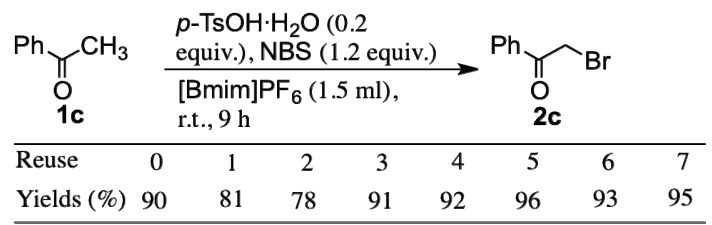


Table 3. Conversion of Ketones into Thiazols with NBS and Thibenzamide in ILs and $\mathrm{CHCl}_{3}$.

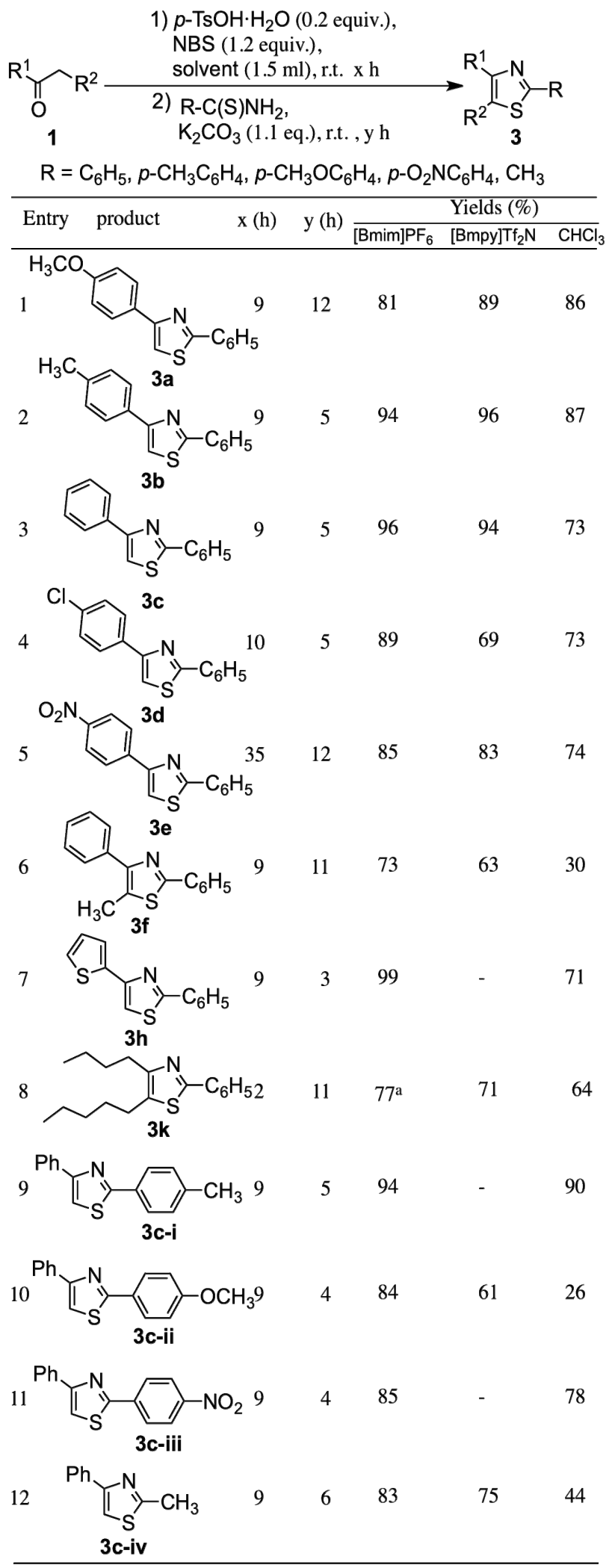

a) Reaction temperature was $50^{\circ} \mathrm{C}$.
Table 4. Recyclic Use of [Bmim]PF6 for preparation of 2,4diphenylthiazol from acetophenone.

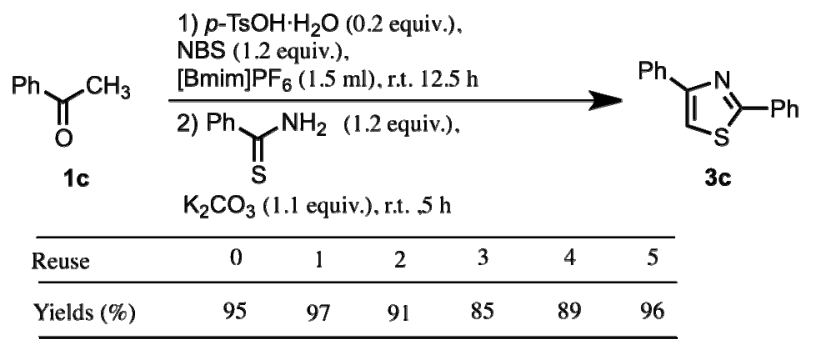

JNM-ECX400, JEOL-JNM-ECS400, and JEOL-JNMECA500 spectrometers. All chemical shifts were expressed in ppm, $\delta$ units down field from TMS $\left(\mathrm{Me}_{4} \mathrm{Si}\right)$. Mass spectra were recorded on JEOL-HX-110 and JEOLJMS-AT15 spectrometers. Melting points were determined on Yamato melting points apparatus Model MP-21. Silica Gel 60 (Kanto Kagaku Co.) and Wakogel B-5F were used for column chromatography and preparative TLC, respectively.

\subsection{Typical Procedure for Conversion of Acetophenone into $p$-bromoacetophenone with NBS and $p$-TsOH $\cdot \mathrm{H}_{2} \mathrm{O}$ in Ionic Liquids}

To a solution of acetophenone $(1 \mathrm{mmol})$ in $\left[\mathrm{Bmim} \mathrm{PF}_{6}\right.$ $(1.5 \mathrm{~mL})$ were added $p$ - $\mathrm{TsOH} \cdot \mathrm{H}_{2} \mathrm{O}(0.2 \mathrm{mmol})$ and NBS (1.2 mmol). The mixture was stirred for $9.5 \mathrm{~h}$ at room temperature. After the reaction, the reaction mixture was extracted with diethyl ether $(10 \mathrm{~mL} \times 7)$. Then, the extract was poured into sat. aq. $\mathrm{Na}_{2} \mathrm{SO}_{3}$ solution. The organic layer was dried over $\mathrm{Na}_{2} \mathrm{SO}_{4}$. After removal of the solvent under reduced pressure, $\alpha$-bromoacetophenone was obtained in the crude state. Purity was estimated by ${ }^{1} \mathrm{H}-\mathrm{NMR}$ to be in the range of $70 \%$ - $80 \%$. Pure $\alpha$-bromoacetophenone was obtained by flash short column chromatography on silica gel $\left(\mathrm{CHCl}_{3}\right.$ :Hexane $\left.=1: 1\right)$ in $90 \%$ yield.

\subsection{Typical Reuse of $[\mathrm{Bmim}] \mathrm{PF}_{6}$}

After the extraction with diethyl ether, the ionic liquid was dried with a vacuum pump for $2 \mathrm{~h}$ at $80^{\circ} \mathrm{C}$. To a solution of acetophenone $(1 \mathrm{mmol})$ in $[\mathrm{Bmim}] \mathrm{PF}_{6}(1.5 \mathrm{~mL})$ were added $p$-TsOH$\cdot \mathrm{H}_{2} \mathrm{O}(0.2 \mathrm{mmol})$ and NBS (1.2 $\mathrm{mmol})$. The mixture was stirred for $9 \mathrm{~h}$ at room temperature. After the reaction, the reaction mixture was extracted with diethyl ether $(10 \mathrm{~mL} \times 7)$. Then, extract was poured into sat. aq. $\mathrm{Na}_{2} \mathrm{SO}_{3}$ solution. The organic layer was dried over $\mathrm{Na}_{2} \mathrm{SO}_{4}$. After removal of the solvent under reduced pressure, $\alpha$-bromoacetophenone was obtained in the crude state. Purity was estimated by ${ }^{1} \mathrm{H}-\mathrm{NMR}$ to be in the range of $70 \%-80 \%$. Pure $\alpha$-bromoacetophenone 
was obtained by flash short column chromatography on silica gel $\left(\mathrm{CHCl}_{3}:\right.$ Hexane $\left.=1: 1\right)$ in $91 \%$ yield.

$\alpha$-Bromoacetophenone: mp $54^{\circ} \mathrm{C}-55^{\circ} \mathrm{C}$ (lit. [81] mp $49^{\circ} \mathrm{C}-50^{\circ} \mathrm{C}$ ); IR(Nujol) 2319, 1690, 1594, 1308, 1276, 1199, 991, 745, $685 \mathrm{~cm}^{-1}$; ${ }^{1} \mathrm{H}$ NMR(500 MHz, $\left.\mathrm{CDCl}_{3}\right): \delta$ $=7.99(\mathrm{~d}, 2 \mathrm{H}, J=7.4 \mathrm{~Hz}, \operatorname{ArH}), 7.62(\mathrm{t}, 1 \mathrm{H}, J=7.4 \mathrm{~Hz}$, $\mathrm{ArH}), 7.50$ (t, 2H, $J=7.4 \mathrm{~Hz}, \mathrm{ArH}), 4.46$ (s, 2H, $-\mathrm{CH}_{2^{-}}$); ${ }^{13} \mathrm{C}$ NMR (100 MHz, $\left.\mathrm{CDCl}_{3}\right): \delta=191.2,133.9$ (3C), 128.9, 128.8, 30.8.

$\alpha$-Bromo-4'-chloroacetophenone: mp $101^{\circ} \mathrm{C}-103^{\circ} \mathrm{C}$ (lit. [82] mp $95^{\circ} \mathrm{C}-96^{\circ} \mathrm{C}$ ); IR(Nujol) 3853, 3749, 3648, 1690, 1540, 1507, 1092, 721, $509 \mathrm{~cm}^{-1}$; ${ }^{1} \mathrm{H}$ NMR(400 $\left.\mathrm{MHz}, \mathrm{CDCl}_{3}\right): \delta=7.99$ (d, 2H, $\left.J=7.3 \mathrm{~Hz}, \mathrm{ArH}\right), 7.62(\mathrm{t}$, $1 \mathrm{H}, J=7.3 \mathrm{~Hz}, \mathrm{ArH}), 7.50$ (t, 2H, $J=8.0 \mathrm{~Hz}, \mathrm{ArH}), 4.46$ (s, $\left.2 \mathrm{H},-\mathrm{CH}_{2} \mathrm{Br}\right) ;{ }^{13} \mathrm{C} \mathrm{NMR}\left(125 \mathrm{MHz}, \mathrm{CDCl}_{3}\right): \delta=190.2$, 140.5, 132.2, 130.3, 129.2, 30.3.

$\alpha$-Bromo-4'-methoxyacetophenone : mp $70^{\circ} \mathrm{C}$ (lit. [83] mp $69^{\circ} \mathrm{C}-73^{\circ} \mathrm{C}$ ); IR(Nujol) 3853, 3749, 3648, 2309, 1683, 1598, 1508, 1322, 1306, 1260, 1205, 1170, 1116, 1020, 986, 840, 816, $721 \mathrm{~cm}^{-1}$; ${ }^{1} \mathrm{H}$ NMR(400 MHz, $\mathrm{CDCl}_{3}$ ): $\delta=7.97$ (d, 2H, $\left.J=7.8 \mathrm{~Hz}, \mathrm{ArH}\right), 6.96$ (d, 2H, $J$ $=7.8 \mathrm{~Hz}, \mathrm{ArH}), 4.40$ (s, $\left.2 \mathrm{H},-\mathrm{CH}_{2^{-}}\right), 3.88\left(\mathrm{~s}, 3 \mathrm{H}, \mathrm{OCH}_{3}\right)$; ${ }^{13} \mathrm{C} \mathrm{NMR}\left(125 \mathrm{MHz}, \mathrm{CDCl}_{3}\right): \delta=189.8,163.9,131.1$, 126.7, 113.9, 55.5, 30.7.

$\alpha$-Bromo-4'-methylacetophenone: mp $56^{\circ} \mathrm{C}-58^{\circ} \mathrm{C}$ (lit. [84] $\mathrm{mp} 48^{\circ} \mathrm{C}-50^{\circ} \mathrm{C}$ ); IR(Nujol) 1687, 1608, 1282, 1179, 799, $723 \mathrm{~cm}^{-1} ;{ }^{1} \mathrm{H} \operatorname{NMR}\left(500 \mathrm{MHz}, \mathrm{CDCl}_{3}\right): \delta=$ 7.88 (d, 2H, $J=8.6 \mathrm{~Hz}, \mathrm{ArH}), 7.28$ (d, $2 \mathrm{H}, J=8.6 \mathrm{~Hz}$, ArH), $4.42\left(\mathrm{~s}, 2 \mathrm{H},-\mathrm{CH}_{2} \mathrm{Br}\right), 2.42\left(\mathrm{~s}, 3 \mathrm{H},-\mathrm{CH}_{3}\right) ;{ }^{13} \mathrm{C}$ NMR (125 MHz, $\left.\mathrm{CDCl}_{3}\right): \delta=190.9,144.9,131.4,129.5$, 129.0, 30.9, 21.7.

$\alpha$-Bromo-4'-nitroacetophenone: mp $98^{\circ} \mathrm{C}-101^{\circ} \mathrm{C}$ (lit. [85] mp $98^{\circ} \mathrm{C}$ ); IR(Nujol) 3853, 3748, 3647, 2309, 1698, 1507, 966, 844, $720 \mathrm{~cm}^{-1}$; ${ }^{1} \mathrm{H}$ NMR (500 MHz, $\left.\mathrm{CDCl}_{3}\right): \delta=8.35$ (d, 2H, $\left.J=8.9 \mathrm{~Hz}, \mathrm{ArH}\right), 8.16$ (d, 2H, $J$ $=8.44 \mathrm{~Hz}, \mathrm{ArH}), 4.46$ (s, 2H, $\left.-\mathrm{CH}_{2} \mathrm{Br}\right) ;{ }^{13} \mathrm{C} \mathrm{NMR}(125$ $\left.\mathrm{MHz}, \mathrm{CDCl}_{3}\right): \delta=189.8,150.7,138.3,130.0,124.0$, 30.1 .

$\alpha$-Bromopropiophenone: Oil; IR(Neat) 3062, 2978, 2925, 1686, 1595, 1448, 1346, 1238, 1160, , 994, 949, $707 \mathrm{~cm}^{-1} ;{ }^{1} \mathrm{H}$ NMR (500 MHz, $\mathrm{CDCl}_{3}$ ): $\delta=8.03$ (d, 2H, $J=7.4 \mathrm{~Hz}, \operatorname{ArH}$ ), 7.59 (t, 1H, $J=6.9 \mathrm{~Hz}, \operatorname{ArH}$ ), 5.29 (q, $J=6.30 \mathrm{~Hz}, 1 \mathrm{H},-\mathrm{CH}-), 1.91$ (d, $J=6.30 \mathrm{~Hz}, 3 \mathrm{H},-\mathrm{CH}_{3}$ ); ${ }^{13} \mathrm{C}$ NMR $\left(125 \mathrm{MHz}, \mathrm{CDCl}_{3}\right): \delta=193.2,134.0,133.6$, 128.8, 128.6, 41.4, 20.0.

$\alpha$-Bromononanophenone: Oil; IR(Neat) 2926, 2855, 1687, 1264, 702, $685 \mathrm{~cm}^{-1} ;{ }^{1} \mathrm{H}$ NMR(500 MHz, $\mathrm{CDCl}_{3}$ ): $\delta=8.01(\mathrm{~d}, 2 \mathrm{H}, J=7.4 \mathrm{~Hz}, \mathrm{ArH}), 7.59(\mathrm{t}, 1 \mathrm{H}, J=7.4 \mathrm{~Hz}$, ArH), 7.48 (t, 2H, $J=7.4 \mathrm{~Hz}, \mathrm{ArH}), 5.13$ (t, $1 \mathrm{H}, J=6.8$ $\mathrm{Hz},-\mathrm{CHBr}-$ ), 2.24-2.07 (m, 2H, $-\mathrm{CH}_{2^{-}}$), 1.53-1.48 (m, 1H, -CH-), 1.43-1.27 (m, 9H, - $\mathrm{CH}_{2-}$ ), 0.89-0.86 (t, 3H, $J$ $\left.=6.8 \mathrm{~Hz},-\mathrm{CH}_{3}\right) ;{ }^{13} \mathrm{C} \mathrm{NMR}\left(125 \mathrm{MHz}, \mathrm{CDCl}_{3}\right): \delta=193.3$, 134.5, 133.6, 128.8, 128.7, 47.3, 33.5, 31.6, 29.1, 29.0, 27.5, 22.5, 14.0 .
2-( $\alpha$-Bromacetyl)thiophene: Oil; IR(Neat) 3544, 3297, 3091, 2942, 2469, 2319, 1660, 1517, 1412, 1355, 1289, 1238, 1193, 1112, 1079, 1061, 1041, 972, 940, 885, 859, 727, 686, 664, 632, $614 \mathrm{~cm}^{-1}$; ${ }^{1} \mathrm{H} \operatorname{NMR}(500 \mathrm{MHz}$, $\left.\mathrm{CDCl}_{3}\right): \delta=7.81$ (d, $1 \mathrm{H}, J=4.0 \mathrm{~Hz}$, thiophene), 7.72 (d, $1 \mathrm{H}, J=4.6 \mathrm{~Hz}$, thiophene), 7.17 (t, $1 \mathrm{H}, J=4.5 \mathrm{~Hz}$, thiophene), 4.36 (s, 2H, $\left.-\mathrm{CH}_{2} \mathrm{Br}\right) ;{ }^{13} \mathrm{C}$ NMR (125 MHz, $\left.\mathrm{CDCl}_{3}\right): \delta=184.3,140.7,135.2$, 133.5, 128.3, 30.5.

$\boldsymbol{\alpha}$-Bromocyclohexanone: Oil; IR(Neat) 2927, 2867, 1715, 1448, 1430, $962 \mathrm{~cm}^{-1} ;{ }^{1} \mathrm{H}$ NMR(400 MHz, $\left.\mathrm{CDCl}_{3}\right)$ : $\delta=4.44(\mathrm{t}, 1 \mathrm{H}, J=5.1 \mathrm{~Hz},-\mathrm{CHBr}-), 3.01-2.95(\mathrm{~m}, 1 \mathrm{H}$, $\left.-\mathrm{CH}_{2^{-}}\right), 2.36$ - $2.29\left(\mathrm{~m}, 2 \mathrm{H},-\mathrm{CH}_{2^{-}}\right), 2.27-2.19(\mathrm{~m}, 1 \mathrm{H}$, $-\mathrm{CH}_{2}-$ ), 2.06 - 1.92 (m, 2H, $-\mathrm{CH}_{2^{-}}$), 1.85 - 1.70 (m, 2H, $\left.-\mathrm{CH}_{2}\right) ;{ }^{13} \mathrm{C}$ NMR (125 MHz, $\left.\mathrm{CDCl}_{3}\right): \delta=203.4,53.4$, 37.9, 36.7, 26.7, 22.1

$\alpha$-Bromocycloheptanone: Oil; IR(Neat) 2933, 2857, 1709, 1454, 1322, 1186, 1159, $935 \mathrm{~cm}^{-1}$; ${ }^{1} \mathrm{H}$ NMR(400 $\mathrm{MHz}, \mathrm{CDCl}_{3}$ ): $\delta=4.38$ (q, 1H, $\left.J=4.6 \mathrm{~Hz},-\mathrm{CHBr}-\right), 2.89$ - 2.82 (m, 1H, -CH-), 2.49 (qd, $1 \mathrm{H}, J=8.0 \mathrm{~Hz}, J=2.96$, -CH-), 2.40 - 2.32 (m, 1H, -CH-), $2.06-1.90$ (m, 3H, -CH-), 1.81 - 1.73 (m, 1H, -CH-), 1.62 - 1.51 (m, 2H, -CH-), 1.43 - 1.34 (m, 1H, -CH-); ${ }^{13} \mathrm{C}$ NMR (100 MHz, $\left.\mathrm{CDCl}_{3}\right): \delta=206.2$, 53.6, 39.3, 34.2, 29.5, 26.7, 24.9.

5-Bromoundecan-6-one: Oil ; IR (Neat) 2958, 2860, 1717, 1464, 1406, 1377, 1241, 1125, 1053, 731; ${ }^{1} \mathrm{H}$ NMR (400 MHz, $\left.\mathrm{CDCl}_{3}\right): \delta=4.23(\mathrm{dd}, 1 \mathrm{H}, J=6.6 \mathrm{~Hz}, J$ $=8.2 \mathrm{~Hz},-\mathrm{CHBr}-), 2.74-2.58\left(\mathrm{~m}, 2 \mathrm{H},-\mathrm{CH}_{2}-\right), 2.04-$ 1.88 (m, 2H, $-\mathrm{CH}_{2^{-}}$), 1.62 (quant, $2 \mathrm{H}, J=7.3 \mathrm{~Hz},-\mathrm{CH}_{2^{-}}$), $2.04-1.27\left(\mathrm{~m}, 8 \mathrm{H},-\mathrm{CH}_{2}^{-}\right), 0.90$ (q, $J=5.5 \mathrm{~Hz}, 6 \mathrm{H}$, $\left.-\mathrm{CH}_{3}\right) ;{ }^{13} \mathrm{C}$ NMR $\left(100 \mathrm{MHz}, \mathrm{CDCl}_{3}\right): \delta=204.4,53.7$, 38.9, 33.1, 31.2, 29.4, 23.6, 22.4, 22.1, 13.9, 13.8.

\subsection{Typical Procedure for Conversion of Acetophenone into 2,4-diphenylthiazole in Ionic Liquid with NBS and Benzthioamide}

To a solution of acetophenone $(1 \mathrm{mmol})$ in $[\mathrm{Bmim}] \mathrm{PF}_{6}$ $(1.5 \mathrm{~mL})$ were added $p$-TsOH$\cdot \mathrm{H}_{2} \mathrm{O}(0.2 \mathrm{mmol})$ and NBS $(1.2 \mathrm{mmol})$. The mixture was stirred for $9 \mathrm{~h}$ at room temperature. Then, benzthioamide $(1.2 \mathrm{mmol})$ and $\mathrm{K}_{2} \mathrm{CO}_{3}$ ( $1.1 \mathrm{mmol})$ were added to the reaction mixture and the obtained mixture was stirred for $5 \mathrm{~h}$ at room temperature. After the reaction, the reaction mixture was extracted with diethyl ether $(10 \mathrm{~mL} \times 10)$. Then, the extract was washed with sat. aq. $\mathrm{Na}_{2} \mathrm{SO}_{3}$ solution. The organic layer was dried over $\mathrm{Na}_{2} \mathrm{SO}_{4}$. After removal of the solvent under reduced pressure, 2,4-diphenylthiazole was obtained in the crude state. Pure 2,4-diphenylthiazol was obtained by flash short column chromatography on silica gel $\left(\mathrm{CHCl}_{3}:\right.$ Hexane $\left.=1: 1\right)$ in $96 \%$ yield.

\subsection{Reuse of $[\mathrm{Bmim}] \mathrm{PF}_{6}$}

After extraction with diethyl ether, the ionic liquid was 
washed with water $(1 \mathrm{~mL})$. The mixture was dried with a vacuum pump for $2 \mathrm{~h}$ at $80^{\circ} \mathrm{C}$. To a solution of acetophenone $(1 \mathrm{mmol})$ in $\left[\mathrm{Bmim}_{\mathrm{P}} \mathrm{PF}_{6}(1.5 \mathrm{~mL})\right.$ were added $p$ - $\mathrm{TsOH} \cdot \mathrm{H}_{2} \mathrm{O}(0.2 \mathrm{mmol})$ and $\mathrm{NBS}(1.2 \mathrm{mmol})$. The mixture was stirred for $12.5 \mathrm{~h}$ at room temperature. Then, benzthioamide and $\mathrm{K}_{2} \mathrm{CO}_{3}(1.1 \mathrm{mmol})$ were added and the obtained mixture was stirred for $5 \mathrm{~h}$ at room temperature. After the reaction, the reaction mixture was extracted with diethyl ether $(10 \mathrm{~mL} \times 10)$. Then, the extract was washed with sat. aq. $\mathrm{Na}_{2} \mathrm{SO}_{3}$ solution. The organic layer was dried over $\mathrm{Na}_{2} \mathrm{SO}_{4}$. After removal of the solvent under reduced pressure, 2,4-diphenylthiazole was obtained in the crude state. Pure 2,4-diphenylthiazole was obtained by flash short column chromatography on silica gel $\left(\mathrm{CHCl}_{3}:\right.$ Hexane $\left.=1: 1\right)$ in $97 \%$ yield

2-Phenyl-4-(4'-methoxyphenyl)thiazole: mp $126^{\circ} \mathrm{C}$ $127^{\circ} \mathrm{C}$ (lit. [86] mp $134^{\circ} \mathrm{C}-135^{\circ} \mathrm{C}$ ). IR(Nujol); 3748, 3648, 2309, 1607, 1520, 1307, 1255, 1172, 1029, 979, 833, 737, $722 \mathrm{~cm}^{-1}$; ${ }^{1} \mathrm{H} \operatorname{NMR}\left(500 \mathrm{MHz}, \mathrm{CDCl}_{3}\right): \delta=$ 8.03 (d, 2H, $J=6.3 \mathrm{~Hz}, \mathrm{ArH}), 7.93$ (d, $2 \mathrm{H}, J=8.6 \mathrm{~Hz}$, $\mathrm{ArH}), 7.45-7.43$ (m, 3H, ArH), 7.34 (s, 1H, thiazole), 6.97 (d, 2H, $J=9.1 \mathrm{~Hz}, \mathrm{ArH}), 3.86$ (s, 3H, $\left.-\mathrm{OCH}_{3}\right) ;{ }^{13} \mathrm{C}$ NMR (125 MHz, $\left.\mathrm{CDCl}_{3}\right): \delta=167.6,159.6,156.1,133.8$, 129.9, 128.8, 127.7, 127.5, 126.5, 114.0, 110.8, 55.3.

2-Phenyl-4-(4'-methylphenyl)thiazole: mp $108^{\circ} \mathrm{C}$ (lit. [86] mp $\left.116^{\circ} \mathrm{C}\right)$; IR(Nujol) 3853, 3749, 3648, 2309, 1698, 1540, 1507, 973, $722 \mathrm{~cm}^{-1} ;{ }^{1} \mathrm{H}$ NMR (500 MHz, $\mathrm{CDCl}_{3}$ ): $\delta=8.04-8.02(\mathrm{~d}, 2 \mathrm{H}, J=6.3 \mathrm{~Hz}, \mathrm{ArH}), 7.89-7.87$ (d, 2H, $J=8.0 \mathrm{~Hz}, \mathrm{ArH}), 7.46-7.41$ (m, 3H, ArH), 7.40 (s, $1 \mathrm{H}$, thiazole), $7.25-7.23$ (d, 2H, $J=6.3 \mathrm{~Hz}, \mathrm{ArH}), 2.39$ (s, 3H, $\left.-\mathrm{CH}_{3}\right) ;{ }^{13} \mathrm{C} \mathrm{NMR}\left(125 \mathrm{MHz}, \mathrm{CDCl}_{3}\right): \delta=167.6$, $156.3,137.9$, 133.8, 131.8, 129.9, 129.3, 128.8, 126.5, 126.3, 111.8, 21.2.

2,4-Diphenylthiazole: $\mathrm{mp} 78^{\circ} \mathrm{C}$ (lit. [87] mp $75^{\circ} \mathrm{C}$ $78^{\circ} \mathrm{C}$ ). IR(Nujol) 760, 725, $465 \mathrm{~cm}^{-1} ;{ }^{1} \mathrm{H} \mathrm{NMR}(500 \mathrm{MHz}$, $\left.\mathrm{CDCl}_{3}\right): \delta=8.05$ (d, $\left.2 \mathrm{H}, J=8.0 \mathrm{~Hz}, \mathrm{ArH}\right), 8.00$ (d, $2 \mathrm{H}, J$ $=8.0 \mathrm{~Hz}, \mathrm{ArH}), 7.49-7.42(\mathrm{~m}, 6 \mathrm{H}$, thiazole, ArH), 7.35 (t, $1 \mathrm{H}, J=7.4 \mathrm{~Hz}, \mathrm{ArH}) ;{ }^{13} \mathrm{C}$ NMR $\left(100 \mathrm{MHz}, \mathrm{CDCl}_{3}\right): \delta$ $=167.8,156.2$, 134.4, 133.7, 130.0, 128.8, 128.7, 128.1, $126.5,126.4,112.5$.

2-Phenyl-4-(4'-chlorophenyl)thiazole: mp $128^{\circ} \mathrm{C}$ (lit. [86] $\left.\mathrm{mp} 131^{\circ} \mathrm{C}-132^{\circ} \mathrm{C}\right)$. IR(Nujol) $1235,1051,766,722$ $\mathrm{cm}^{-1} ;{ }^{1} \mathrm{H} \mathrm{NMR}\left(500 \mathrm{MHz}, \mathrm{CDCl}_{3}\right): \delta=8.03$ (d, 2H, $J=$ $8.0 \mathrm{~Hz}, \mathrm{ArH}), 7.93$ (d, 2H, $J=8.5 \mathrm{~Hz}, \mathrm{ArH}), 7.48-7.44$ (m, 4H, ArH, thiazole), 7.41 (d, 2H, $J=8.5 \mathrm{~Hz}, \mathrm{ArH})$; ${ }^{13} \mathrm{C}$ NMR (125 MHz, $\mathrm{CDCl}_{3}$ ): $\delta=168.1,155.0,133.9$, 133.5, 132.9, 130.1, 128.9, 128.8, 127.6, 126.5,112.8.

2-Phenyl-4-(4'-nitrophenyl)thiazole: mp $125^{\circ} \mathrm{C}$ $127^{\circ} \mathrm{C}$ (lit. [88] mp $122^{\circ} \mathrm{C}$ ); IR(Nujol) 1597, 1509, 1341, 1058, 974, 842, 734, $665 \mathrm{~cm}^{-1}$; ${ }^{1} \mathrm{H}$ NMR (400 MHz, $\left.\mathrm{CDCl}_{3}\right): \delta=8.31$ (d, $\left.2 \mathrm{H}, J=8.8 \mathrm{~Hz}, \mathrm{ArH}\right), 8.17$ (d, $2 \mathrm{H}, J$ $=8.8 \mathrm{~Hz}, \mathrm{ArH}), 8.06-8.03(\mathrm{~m}, 2 \mathrm{H}, \mathrm{ArH}), 7.69(\mathrm{~s}, 1 \mathrm{H}$, thiazole), 7.52 - 7.48 (m, 3H, ArH); ${ }^{13} \mathrm{C}$ NMR (125 MHz,
$\left.\mathrm{CDCl}_{3}\right): \delta=168.7,153.7,147.2,140.2,133.1,130.5$, 129.0, 126.9, 126.6, 124.1, 115.9.

2,4-Diphenyl-5-methylthiazole: $\mathrm{mp} 75^{\circ} \mathrm{C}-76^{\circ} \mathrm{C}$ (lit. [89] $\mathrm{mp} 76^{\circ} \mathrm{C}$ ); IR(Nujol) 2723, 1306, 970, 760, 721, 690 $\mathrm{cm}^{-1} ;{ }^{1} \mathrm{H}$ NMR(400 MHz, $\left.\mathrm{CDCl}_{3}\right): \delta=7.96(\mathrm{~d}, 2 \mathrm{H}, J=$ $6.4 \mathrm{~Hz}, \mathrm{ArH}), 7.73$ (d, 2H, $J=6.8 \mathrm{~Hz}, \mathrm{ArH}), 7.48$ - 7.39 (m, 6H, ArH, thiazole), 2.61 (s, 3H, $\left.-\mathrm{CH}_{3}\right) ;{ }^{13} \mathrm{C} \mathrm{NMR}$ $\left(100 \mathrm{MHz}, \mathrm{CDCl}_{3}\right): \delta=163.5,151.9,135.1,133.8,129.5$, 128.7, 128.6, 128.3, 127.5, 126.2, 12.8.

2-Phenyl-4-(2'-thienyl)thiazole: mp 58 - 60 (lit. [90] mp $69^{\circ} \mathrm{C}-71^{\circ} \mathrm{C}$ ); IR(Nujol) 1664, 1024, 970, 763, 691, $740 \mathrm{~cm}^{-1} ;{ }^{1} \mathrm{H}$ NMR(400 MHz, $\left.\mathrm{CDCl}_{3}\right): \delta=8.04-8.01$ (m, 2H, ArH), 7.53 (dd, $1 \mathrm{H}, J=3.6 \mathrm{~Hz}, J=1.1 \mathrm{~Hz}$, thienyl), 7.49 - 7.45 (m, 3H, ArH), 7.35 (s, 1H, thiazol), 7.32 (dd, $1 \mathrm{H}, J=5.0 \mathrm{~Hz}, J=1.1 \mathrm{~Hz}$, thienyl), 7.10 (dd, $1 \mathrm{H}, J=3.6 \mathrm{~Hz}, J=5.2 \mathrm{~Hz}$, thienyl); ${ }^{13} \mathrm{C}$ NMR $(125 \mathrm{MHz}$, $\left.\mathrm{CDCl}_{3}\right): \delta=167.9,150.7,138.3,133.3,130.1,128.9$, 127.6, 126.6, 125.3, 124.2, 111.3.

4-Butyl-2-phenyl-5-pentylthiazole: Oil. IR (neat) 2928, 2857, 1536, 1461, 1248, 991, 760, $689 \mathrm{~cm}^{-1}$; ${ }^{1} \mathrm{H}$ NMR (400 MHz, $\left.\mathrm{CDCl}_{3}\right): \delta=7.87(\mathrm{~d}, 2 \mathrm{H}, J=6.6 \mathrm{~Hz}, \mathrm{ArH})$, 7.41-7.33 (m, 3H, ArH), 2.75 (t, 2H, $J=7.5 \mathrm{~Hz},-\mathrm{CH}_{2}-$ ), 2.68 (t, $2 \mathrm{H}, J=7.5 \mathrm{~Hz},-\mathrm{CH}_{2}-$ ), 1.71 (quant, $2 \mathrm{H}, J=7.5$ $\mathrm{Hz},-\mathrm{CH}_{2}-$ ), 1.63 (quant, $2 \mathrm{H}, J=7.8 \mathrm{~Hz},-\mathrm{CH}_{2}-$ ), $1.46-$ $1.33\left(\mathrm{~m}, 6 \mathrm{H},-\mathrm{CH}_{2}-\right), 0.95$ (t, 3H, $\left.J=7.3 \mathrm{~Hz},-\mathrm{CH}_{3}\right), 0.90$ (t, 3H, $\left.\left.J=7.1 \mathrm{~Hz},-\mathrm{CH}_{3}\right) ;{ }^{13} \mathrm{C} \mathrm{NMR} \mathrm{(125} \mathrm{MHz,} \mathrm{CDCl}_{3}\right)$ : $\delta=163.5,153.4,134.2,132.7,129.2,128.7,126.1,34.2$, 31.7, 29.6, 29.2, 26.1, 22.5, 22.2, 14.0, 13.8; HRMS Calcd for $\mathrm{C}_{18} \mathrm{H}_{26} \mathrm{NS}$ 288.1780, Found; 288.1774.

4-Phenyl-2-(4'-methylphenyl)thiazole: mp $120^{\circ} \mathrm{C}$ $122^{\circ} \mathrm{C}$ (lit. [91] mp $127^{\circ} \mathrm{C}-128^{\circ} \mathrm{C}$ ); IR(Nujol) 1056, 972, 814, 739, $689 \mathrm{~cm}^{-1} ;{ }^{1} \mathrm{H} \operatorname{NMR}\left(400 \mathrm{MHz}, \mathrm{CDCl}_{3}\right): \delta=$ 7.99 (d, 2H, $J=7.3 \mathrm{~Hz}, \operatorname{ArH}), 7.93$ (d, 2H, $J=8.2 \mathrm{~Hz}$, ArH), 7.46 - 7.42 (m, 2H, ArH, thiazol), 7.34 (t, 1H, $J=$ $7.3 \mathrm{~Hz}, \mathrm{ArH}) 7.26$ (d, $1 \mathrm{H}, J=6.4 \mathrm{~Hz}, \mathrm{ArH}) 2.41$ (s, 3H, $\left.-\mathrm{CH}_{3}\right) ;{ }^{13} \mathrm{C}$ NMR $\left(125 \mathrm{MHz}, \mathrm{CDCl}_{3}\right): \delta=168.0,156.1$, 140.2 , 134.6, 131.1, 129.5, 128.6, 128.0, 126.5, 126.4, 112.1, 21.4 .

4-Phenyl-2-(4'-methoxyphenyl)thiazole: $\mathrm{mp} 96^{\circ} \mathrm{C}$ $98^{\circ} \mathrm{C}$ (lit. [91] mp $\left.101^{\circ} \mathrm{C}\right)$; IR(Nujol) 1519, 1254, 979, 833, $737 \mathrm{~cm}^{-1}$; ${ }^{1} \mathrm{H} \mathrm{NMR}\left(400 \mathrm{MHz}, \mathrm{CDCl}_{3}\right): \delta=7.98(\mathrm{~d}$, $2 \mathrm{H}, J=9.1 \mathrm{~Hz}, \mathrm{ArH}), 7.44$ (t, 2H, $J=7.3 \mathrm{~Hz}, \mathrm{ArH}), 7.41$ (s, $1 \mathrm{H}$, thiazole), 7.34 (t, $1 \mathrm{H}, J=7.3 \mathrm{~Hz}, \mathrm{ArH}), 3.86$ (s, $\left.3 \mathrm{H},-\mathrm{OCH}_{3}\right) ;{ }^{13} \mathrm{C} \mathrm{NMR}\left(125 \mathrm{MHz}, \mathrm{CDCl}_{3}\right): \delta=167.6$, $161.1,155.9$, 134.6, 128.6, 128.0, 126.7, 126.3, 114.2, $111.7,55.3$.

2-(4'-Nitrophenyl)-4-Phenylthiazol: $\mathrm{mp} 162^{\circ} \mathrm{C}-164^{\circ} \mathrm{C}$ (lit. [91] mp $164^{\circ} \mathrm{C}-165^{\circ} \mathrm{C}$ ); IR(Nujol) $1595,1512,1340$, 848, 751, 722, $687 \mathrm{~cm}^{-1} ;{ }^{1} \mathrm{H} \mathrm{NMR}\left(500 \mathrm{MHz}, \mathrm{CDCl}_{3}\right): \delta$ $=8.33(\mathrm{~d}, 2 \mathrm{H}, J=9.2 \mathrm{~Hz}, \operatorname{ArH}), 8.22(\mathrm{~d}, 2 \mathrm{H}, J=9.2 \mathrm{~Hz}$, ArH), 8.00 (d, 2H, $J=6.8 \mathrm{~Hz}, \mathrm{ArH}), 7.62$ (s, 1H, thiazol), 7.47 (t, $1 \mathrm{H}, J=7.4 \mathrm{~Hz}, \operatorname{ArH}), 7.39$ (t, $1 \mathrm{H}, J=7.4 \mathrm{~Hz}$, $\mathrm{ArH}) ;{ }^{13} \mathrm{C} \mathrm{NMR}\left(100 \mathrm{MHz}, \mathrm{CDCl}_{3}\right): \delta=164.7,157.3$, 
148.4, 139.1, 133.8, 128.8, 128.6, 127.1, 126.4, 124.3, 114.5 .

2-Methyl-4-phenylthiazole: $\mathrm{mp} 64^{\circ} \mathrm{C}$ (lit. [92] $\mathrm{mp}$ $64^{\circ} \mathrm{C}$ ); IR(Nujol) 740, 726, 692, $675 \mathrm{~cm}^{-1}$; ${ }^{1} \mathrm{H}$ NMR(400 $\mathrm{MHz}_{\mathrm{CDCl}}$ ): $\delta=7.87$ (d, 2H, $\left.J=6.8 \mathrm{~Hz}, \mathrm{ArH}\right), 7.41$ (t, $2 \mathrm{H}, J=7.8 \mathrm{~Hz}, \mathrm{ArH}), 7.33-7.30$ (m, 2H, ArH, thiazole), 2.78 (s, 3H, $\left.\left.\mathrm{CH}_{3}\right) ;{ }^{13} \mathrm{C} \mathrm{NMR} \mathrm{(125} \mathrm{MHz,} \mathrm{CDCl}_{3}\right): \delta=$ 165.7, 155.1, 134.5, 128.6, 127.9, 126.2, 112.2, 19.3.

\section{Acknowledgements}

Financial support in the form of a Grant-in-Aid for Scientific Research (No. 20550033) from the Ministry of Education, Culture, Sports, Science, and Technology in Japan, and Iodine Research Project in Chiba University is gratefully acknowledged.

\section{References}

[1] A. Benazzouz, T. Boraud, P. Dubedat, A. Boireau, J. M. Stutzmann and C. Gross, "Riluzole Prevents MPTP-Induced Parkinsonism in the Rhesus Monkey: A Pilot Study," European Journal of Pharmacology, Vol. 284, No. 3, 1995, pp. 299-307. doi:10.1016/0014-2999(95)00362-O

[2] A. M. Liberatore, J. Schultz, C. Favre-Guilmard, J. Pommier, P. E. Chabrier and D. Bigg, "Butyl 2-(4-[1.1'Biphenyl]-4-yl-1H-Imidazol-2-yl)ethylcarbamate, a Potent Sodium Channel Blocker for the Treatment of Neuropathic Pain,” Bioorganic \& Medicinal Chemistry Letters, Vol. 17, No. 6, 2007, pp. 1746-1749. doi:10.1016/j.bmcl.2006.12.064

[3] F. Haviv, J. D. Ratajczyk, R. W. DeNet, F. A. Kerdesky, R. L. Walters, S. P. Schmidt, J. H. Holms, P. R. Young and G. W. Carter, "3-[1-(2-Benzoxazolyl)hydrazino] Propanenitrile Derivatives: Inhibitors of Immune Complex Induced Inflammation,” Journal of Medicinal Chemistry, Vol. 31, No. 9, 1988, pp. 1719-1728.

doi:10.1021/jm00117a010

[4] F. Clemence, O. L. Marter, F. Delevalle, J. Benzoni, A. Jouanen, S. Jouquey, M. Mouren and R. Deraedt, “4-Hydroxy-3-Quinolinecarboxamides with Antiarthritic and Analgesic Activities,” Journal of Medicinal Chemistry, Vol. 31, No. 7, 1988, pp. 1453-1462. doi:10.1021/jm00402a034

[5] F. W. Bell, A. S. Cantrell, M. Hogberg, S. R. Jaskunas, N. G. Johansson, C. L. Jordon, M. D. Kinnick, P. Lind, J. M. Morin Jr, R. Noreen, B. Oberg, J. A. Palkowitz, C. A. Parrish, P. Pranc, C. Sahlberg, R. J. Ternansky, R. T. Vasileff, L. Vrang, S. J. West, H. Zhang and X. X. Zhou, "Phenethylthiazolethiourea (PETT) Compounds, a New Class of HIV-1 Reverse Transcriptase Inhibitors. 1. Synthesis and Basic Structure-Activity Relationship Studies of PETT Analogs," Journal of Medicinal Chemistry, Vol. 38, No. 25, 1995, pp. 4929-4936. doi: $10.1021 / \mathrm{jm} 00025 \mathrm{a} 010$

[6] K. Tsuji and H. Ishikawa, "Synthesis and Anti-Pseudo- monal Activity of New 2-Isocephems with a Dihydroxypyridone Moiety at C-7," Bioorganic \& Medicinal Chemistry Letters, Vol. 4, No. 13, 1994, pp. 1661-1606. doi:10.1016/S0960-894X(01)80574-6

[7] X. H. Gu, X. Z. Wan and B. Jiang, "Syntheses and Biological Activities of Bis(3-Indolyl)thiazoles, Analogues of Marine Bis(Indole)alkaloid Nortopsentins," Bioorganic \& Medicinal Chemistry Letters, Vol. 9, No. 4, 1999, pp. 569-572. doi:10.1016/S0960-894X(99)00037-2

[8] T. David, P. Enrico and X. Zhunjie, “One-Pot Synthesis of New 2,4,5-Trisubstituted 1,3-Thiazoles and 1,3-Selenazoles,” Tetrahedron, Vol. 65, No. 15, 2009, pp. 29822988. doi:10.1016/j.tet.2009.01.104

[9] X. Zhang, W. T. Teo, S. Chan and P. Chan, "Brønsted Acid Catalyzed Cyclization of Propargylic Alcohols with Thioamides. Facile Synthesis of Di- and Tri-substituted Thiazoles," The Journal of Organic Chemistry, Vol. 75, No. 18, 2010, pp. 6290-6293. doi:10.1021/jo101292r

[10] K. M. Weiss, S. Wei and S. Tsogoera, "Novel One-Pot Process for the Synthesis of 1,3-Thiazoles via Organocatalysed Epoxidation of Nitro-Olefins," Organic \& Biomolecular Chemistry, Vol. 9, No. 9, 2011, pp. 3457-3461. doi:10.1039/c1ob05260h

[11] E. K. Said, B. Sabine and M. Adberrahim, "Solid-Phase Synthesis of Imidazo[1,2-a]pyridines and Imidazo[1,2-a] pyrimidines," Tetrahedron Letters, Vol. 44, No. 33, 2003, pp. 6265-6267. doi:10.1016/S0040-4039(03)01532-6

[12] A. Mounir, M. Emmanuel, C. Oliver and C. T. Jean, “A Convenient Synthesis of Linear Pyridinoimidazo[1,2-a] pyridine and Pyrroloimidazo[1,2-a]Pyridine cores," Tetrahedron Letters, Vol. 48, No. 47, 2007, pp. 8392-8395. doi:10.1016/j.tetlet.2007.07.223

[13] A. Hantzsch and J. H. Weber, "Ueber Verbindungen des Thiazols (Pyridins der Thiophenreihe)," Berichte der Deutschen Chemischen Gesellschaft, Vol. 20, No. 2, 1887, pp. 3118- 3132.

[14] R. H. Wiley and L. C. Behr, “Organic Reactions,” Vol. 6, Wiley, New York, 1951, pp. 367-409.

[15] G. K. S. Prakash, R. Ismail, J. Garcia, C. Panja, G. Rasul, T. Mathew and G. A. Olah, " $\alpha$-Halogenation of Carbonyl Compounds: Halotrimethylsilane-Nitrate Salt Couple as an Efficient Halogenating Reagent System,” Tetrahedron Letters, Vol. 5, No. 11, 2011, pp. 1217-1221. doi:10.1016/j.tetlet.2011.01.039

[16] R. M. Moriarty, R. K. Vaid and G. F. Koser, "[Hydroxy (organosulfonyloxy)iodo]arenes in Organic Synthesis," Synlett, Vol. 7, 1990, pp. 365-383. doi:10.1055/s-1990-21097

[17] G. F. Koser, Aldrichimica, 2001, 34, 89-102. papers:

[18] T. Muraki, H. Togo and M. Yokoyama, "Reactivity and Synthetic Utility of 1-(Arenesulfonyloxy)benziodoxolones,” The Journal of Organic Chemistry, Vol. 64, No. 8, 1999, pp. 2883-2889. doi:10.1021/jo9825207

[19] T. Nabana and H. Togo, "Reactivities of Novel [Hydroxy(tosyloxy)iodo]arenes and [Hydroxy(phosphoryloxy)iodo]arenes for $\alpha$-Tosyloxylation and $\alpha$-Phosphoryloxylation of Ketones," The Journal of Organic Chemistry, 
Vol. 67, No. 12, 2002, pp. 4362-4365. doi:10.1021/jo0200670

[20] Y. Misu and H. Togo, "Novel Preparation of 2,1-Benzothiazine Derivatives from Sulfonamides with [Hydroxy(tosyloxy)iodo]arenes," Organic \& Biomolecular Chemistry, Vol. 1, No. 8, 2003, pp. 1342-1346. doi:10.1039/b301330h

[21] M. Ueno, T. Nabana and H. Togo, "Novel Oxidative $\alpha$-Tosyloxylation of Alcohols with Iodosylbenzene and $p$-Toluenesulfonic Acid and Its Synthetic Use for Direct Preparation of Heteroaromatics," The Journal of Organic Chemistry, Vol. 68, No. 16, 2003, pp. 6424-6426.

[22] D. Kumar, N. M. Kumar, G. Patel, S. Gupta and R. S. Varma, "A Facile and Eco-friendly Synthesis of Diarylthiazoles and Diarylimidazoles in Water," Tetrahedron Letters, Vol. 52, No. 16, 2011, pp. 1983-1986.

[23] T. Welton, "Room-Temperature Ionic Liquids. Solvents for Synthesis and Catalysis," Chemical Reviews, Vol. 99, No. 8, 1999, pp. 2071-2084. doi:10.1021/cr980032t

[24] P. Wasserscheid and W. Keim, "Ionic Liquids-New 'Solutions' for Transition Metal Catalysis," Angewandte Chemie International Edition, Vol. 39, No. 21, 2000, pp. 3772-3789.

doi:10.1002/1521-3773(20001103)39:21<3772::AID-AN IE3772>3.0.CO;2-5

[25] R. Sheldon, "Catalytic Reactions in Ionic Liquids," Chemical Communications, No. 23, 2001, pp. 2399-2407. doi:10.1039/b107270f

[26] R. A. Sheldon, "Atom Efficiency and Catalysis in Organic Synthesis,” Pure Applied Chemistry, Vol. 72, No. 7, 2001, pp. 1233-1246. doi:10.1351/pac200072071233

[27] M. J. Earle and K. R. Seddon, "Ionic Liquids. Green Solvents for the Future,” Pure Applied Chemistry, Vol. 72, No. 7, 2000, pp. 1391-1398. doi:10.1351/pac200072071391

[28] H. Zhao, et al., "Poly(Isonicotinic Acid) Modified Glassy Carbon Electrode for Electrochemical Detection of Norepinephrine,” Analytica Chimica Acta, Vol. 454, No. 1, 2002, pp. 75-81. doi:10.1016/S0003-2670(01)01543-4

[29] S. Lee, "Functionalized Imidazolium Salts for Task-Specific Ionic Liquids and Their Applications,” Chemical Communications, No. 10, 2006, pp. 1049-1063. doi:10.1039/b514140k

[30] D. R. Macfarlane, J. M. Pringle, K. M. Johansson, S. A. Forsyth and M. Forsyth, "Lewis Base Ionic Liquids," Chemical Communications, No. 18, 2006, pp. 1905-1917.

[31] J. K. D. Surette, L. Green and R. D. Singer, "1-Ethyl-3Methylimidazolium Halogenoaluminate Melts as Reaction Media for the Friedel-Crafts Acylation of Ferrocene," Chemical Communications, No. 24, 2006, pp. 2753-2754. doi:10.1039/cc9960002753

[32] C. J. Adams, M. J. Earle, G. Roberts, K. R. Seddon, Chem. Commun. 1998, 2097-2098.

[33] Y. R. Jorapur, C. H. Lee, D. and Y. Chi, "Mono- and Dialkylations of Pyrrole at C2 and C5 Positions by Nucleophilic Substitution Reaction in Ionic Liquid,” Or- ganic Letters, Vol. 7, No. 7, 2005, pp. 1231-1254. doi:10.1021/ol047446v

[34] A. L. Monteiro, F. K. Zinn, R. F. De Souza and J. Dupont, "Asymmetric Hydrogenation of 2-Arylacrylic Acids Catalyzed by Immobilized Ru-BINAP Complex in 1- $n$ Butyl-3-Methy- limidazolium Tetrafluoroborate Molten Salt," Tetrahedron: Asymmetry, Vol. 8, No. 2, 1997, pp. 177-179. doi:10.1016/S0957-4166(96)00485-5

[35] P. J. Dyson, D. L. Ellis, D. G. Parker and T. Welton, "Arene Hydrogenation in a Room-Temperature Ionic Liquid Using a Ruthenium Cluster Catalyst,” Chemical Communications, No. 1, 1999, pp. 25-26. doi:10.1039/a807447j

[36] C. J. Adams, M. J. Earle and K. R. Seddon, "Stereoselective Hydrogenation Reactions in Chloroaluminate(III) Ionic Liquids: A New Method for the Reduction of Aromatic Compounds," Chemical Communications, No. 11, 1999, pp. 1043-1044. doi:10.1039/a901302d

[37] J. Howarth, K. Hanlon, D. Fayne and P. McCormac, "Moisture Stable Dialkylimidazolium Salts as Heterogeneous and Homogeneous Lewis Acids in the Diels-Alder Reaction,” Tetrahedron Letters, Vol. 38, No. 17, 1997, pp. 3097-3100. doi:10.1016/S0040-4039(97)00554-6

[38] J. G. Huddleston and R. D. Rogers, "Room Temperature Ionic Liquids as Novel Media for 'Clean’ Liquid-Liquid Extraction,” Chemical Communications, No. 16, 1998, pp. 1765-1766. doi:10.1039/a803999b

[39] C. W. Lee, "Diels-Alder Reactions in Chloroaluminate Ionic Liquids: Acceleration and Selectivity Enhancement," Tetrahedron Letters, Vol. 40, No. 13, 1999, pp. 2461-2464. doi:10.1016/S0040-4039(99)00249-X

[40] A. J. Carmichael, M. J. Earle, J. D. Holbrey, P. B. McCormac and K. R. Seddon, "The Heck Reaction in Ionic Liquids: A Multiphasic Catalyst System,” Organic Letters, Vol. 1, No. 7, 1999, pp. 997-1000. doi:10.1021/ol9907771

[41] V. Calo, A. Nacci, L. Lopez and N. Mannarini, "Heck Reaction in Ionic Liquids Catalyzed by a Pd-Benzothiazole Carbene Complex," Tetrahedron Letters, Vol. 41, No. 46, 2000, pp. 8973-8976. doi:10.1016/S0040-4039(00)01592-6

[42] C. J. Mathews, P. J. Smith and T. Welton, "Palladium Catalysed Suzuki Cross-Coupling Reactions in Ambient Temperature Ionic Liquids,” Chemical Communications, No. 14, 2000, pp. 1249-1250. doi:10.1039/b002755n

[43] T. Fukuyama, M. Shinmen, S. Nishitani, M. Sato and I. Ryu, "A Copper-Free Sonogashira Coupling Reaction in Ionic Liquids and Its Application to a Microflow System for Efficient Catalyst Recycling,” Organic Letters, Vol. 4, No. 10, 2002, pp. 1691-1694. doi:10.1021/ol0257732

[44] K. G. Mayo, E. H. Nearhoof and J. J. Kiddle, "Microwave-Accelerated Ruthenium-Catalyzed Olefin Metathesis,” Organic Letters, Vol. 4, No. 9, 2002, pp. 1567-1570. doi:10.1021/ol025789s

[45] V. Calo, A. Nacci, L. Lopez and V. L. Lerario, "Stereoselective Synthesis of Electrophilic Spirocyclopropanes in Ionic Liquids," Tetrahedron Letters, Vol. 41, No. 46, 2000, pp. 8977-8980. 


\section{doi:10.1016/S0040-4039(00)01593-8}

[46] G. S. Owens and M. M. Abu-Omar, "Methyltrioxorhenium-Catalyzed Epoxidations in Ionic Liquids," Chemical Communications, No. 13, 2000, pp. 1165-1166. doi:10.1039/b001661f

[47] J. Howarth, "Oxidation of Aromatic Aldehydes in the Ionic Liquid [Bmim] $\mathrm{PF}_{6}$," Tetrahedron Letters, Vol. 41, No. 34, 2000, pp. 6627-6629. doi:10.1016/S0040-4039(00)01037-6

[48] I. A. Ansari and R. Gree, "TEMPO-Catalyzed Aerobic Oxidation of Alcohols to Aldehydes and Ketones in Ionic Liquid [Bmim] $\left[\mathrm{PF}_{6}\right]$," Organic Letters, Vol. 4, No. 9, 2002, pp. 1507-1509. doi:10.1021/ol025721c

[49] R. Yanada and Y. Takemoto, “ $\mathrm{OsO}_{4}$-Catalyzed Dihydroxylation of Olefins in Ionic Liquid [Emim]BF 4 : A Recoverable and Reusable Osmium," Tetrahedron Letters, Vol. 43, No. 38, 2002, pp. 6849-6852. doi:10.1016/S0040-4039(02)01461-2

[50] Z. Liu, Z. C. Chen and Q. G. Zheng, "Mild Oxidation of Alcohols with O-Iodoxybenzoic Acid (IBX) in Ionic Liquid 1-Butyl-3-Methyl-Imidazolium Chloride and Water," Organic Letters, Vol. 5, No. 18, 2003, pp. 3321-3324. doi:10.1021/ol0351549

[51] J. S. Yadav, B. V. S. Reddy, A. K. Basak and A. V. Narsaiah, "Recyclable 2nd Generation Ionic Liquids as Green Solvents for the Oxidation of Alcohols with Hypervalent Iodine Reagents,” Tetrahedron, Vol. 60, No. 9, 2004, pp. 2131-2135. doi:10.1016/j.tet.2003.12.056

[52] B. S. Chhikara, S. Tehlan, A. Kumar, Synlett 2005, 63-66.

[53] X. E. Wu, L. Ma, M. X. Ding and L. X. Gao, "Imidazolium Ionic Liquid-Grafted 2,2'-Bipyridine-A Novel Ligand for the Recyclable Copper-catalyzed Selective Oxidation of Alcohols in Ionic Liquid [bmim] $\left[\mathrm{PF}_{6}\right]$," Chemistry Letters, Vol. 34, No. 3, 2005, pp. 312-313. doi:10.1246/cl.2005.312

[54] X. E. Wu, L. Ma, M. X. Ding and L. X. Gao, "TEMPO-Derived Task-Specific Ionic Liquids for Oxidation of Alcohols,” ChemInform, Vol. 36, No. 32, 2005, pp. 607-610.

[55] D. W. Morrison, D. C. Forbes and J. H. Davis Jr, "Base-Promoted Reactions in Ionic Liquid Solvents. The Knoevenagel and Robinson Annulation Reactions," Tetrahedron Letters, Vol. 42, No. 35, 2001, pp. 6053-6056. doi:10.1016/S0040-4039(01)01228-X

[56] Y. Y. Xie, Z. C. Chen and Q. G. Zheng, "Organic Reactions in Ionic Liquids: Ionic Liquid-Accelerated Cyclocondensation of $\alpha$-Tosyloxyketones with 2-Aminopyridine,” ChemInform, Vol. 33, No. 50, 2002, pp. 111- 114.

[57] C. Su, Z. C. Chen and Q. G. Zheng, “Organic Reactions in Ionic Liquids: Knoevenagel Condensation Catalyzed by Ethylenediammonium Diacetate," Synthesis, Vol. 0, No. 4, 2003, pp. 555-559.

[58] S. Kitaoka, K. Nobuoka and Y. Ishikawa, "The First Utilization of Acidic Ionic Liquid for Preparation of Tetraarylporphyrins," Chemical Communications, No. 17, 2004, pp. 1902-1903. doi:10.1039/b404241g
[59] A. Sato, Y. Nakamura, T. Maki, K. Ishihara and A. Yamamoto, “Zr(IV), Fe(III), Ga(III), and Sn(IV) Binary Metal Complexes as Synergistic and Reusable Esterification Catalysts," Advanced Synthesis \& Catalysis, Vol. 347, No. 10, 2005, pp. 1337-1340. doi:10.1002/adsc.200505083

[60] C. K. Z. Andrade, S. C. S. Takeda, L. M. Alves, J. P. Rodrigues, P. A. Z. Suarez, R. F. Brandao and V. C. D. Soares, "Molecular Sieves in Ionic Liquids as an Efficient and Recyclable Medium for the Synthesis of Imines," Synlett, Vol. 12, 2004, pp. 2135-2138. doi:10.1055/s-2004-831328

[61] R. X. Ren, L. D. Zueva and W. Ou, "Formation of $\varepsilon$-Caprolactam via Catalytic Beckmann Rearrangement Using $\mathrm{P}_{2} \mathrm{O}_{5}$ in Ionic Liquids," Tetrahedron Letters, Vol. 42, No. 48, 2001, pp. 8441-8443. doi:10.1016/S0040-4039(01)01850-0

[62] Y. Deng, F. Shi, J. Beng and K. Quio, "Ionic Liquid as a Green Catalytic Reaction Medium for Esterifications," Journal of Molecular Catalysis A: Chemical, Vol. 165, No. 1-2, 2001, pp. 33-36. doi:10.1016/S1381-1169(00)00422-2

[63] J. Fraga-Dubreuil, K. Bourahla, M. Rahmouni, J. P. Bazureau and J. Hamelin, "Catalysed Esterifications in Room Temperature Ionic Liquids with Acidic Counteranion as Recyclable Reaction Media," Catalysis Communications, Vol. 3, No. 5, 2002, pp. 185-190. doi:10.1016/S1566-7367(02)00087-0

[64] L. Brinchi, R. Germani, G. Savelli, "Ionic Liquids as Reaction Media for Esterification of Carboxylate Sodium Salts with Alkyl Halides,” Tetrahedron Letters, Vol. 44, No. 10, 2003, pp. 2027-2029. doi:10.1016/S0040-4039(03)00179-5

[65] J. McNulty, S. Cheekoori, J. J. Nair, V. Larichev, A. Capretta and A. J. Robertson, "A Mild Esterification Process in Phosphonium Salt Ionic Liquid,” Tetrahedron Letters, Vol. 46, No. 21, 2005, pp. 3641-3644. doi:10.1016/j.tetlet.2005.03.169

[66] D. W. Kim, C. E. Song and D. Y. Chi, "New Method of Fluorination Using Potassium Fluoride in Ionic Liquid: Significantly Enhanced Reactivity of Fluoride and Improved Selectivity," Journal of the American Chemical Society, Vol. 124, No. 35, 2002, pp. 10278-10279. doi:10.1021/ja026242b

[67] C. Chiappe, D. Pieraccini and P. Saullo, "Nucleophilic Displacement Reactions in Ionic Liquids: Substrate and Solvent Effect in the Reaction of NaN3 and KCN with Alkyl Halides and Tosylates," Journal of the American Chemical Society, Vol. 68, No. 17, 2003, pp. 6710-6715. doi:10.1021/jo026838h

[68] L. Brinchi, R. Germani and G. Savelli, "Efficient Esterification of Carboxylic Acids with Alkyl Halides Catalyzed by Fluoride Ions in Ionic Liquids," Tetrahedron Letters, Vol. 44, No. 35, 2003, pp. 6583-6585. doi:10.1016/S0040-4039(03)01693-9

[69] L. Brinchi, R. Germani and G. Savelli, "Ionic Liquids as Reaction Media for Esterification of Carboxylate Sodium Salts with Alkyl Halides," Tetrahedron Letters, Vol. 44, No. 10, 2003, pp. 2027-2029. 


\section{doi:10.1016/S0040-4039(03)00179-5}

[70] S. S. Mohile, M. K. Potdar and M. M. Salunkhe, “An Ionic Liquid-Mediated Expeditious Route to the Syntheses of Diaryl Sulfoxides,” Tetrahedron Letters, Vol. 44, No. 6, 2003, pp. 1255-1258. doi:10.1016/S0040-4039(02)02786-7

[71] J. S. Yadav, B. V. S. Reddy, A. K. Basak and A. V. Narsaiah, "[Bmim] $\mathrm{PF}_{6}$ and $\mathrm{BF}_{4}$ Ionic Liquids as Novel and Recyclable Reaction Media for Aromatic Amination," Tetrahedron Letters, Vol. 44, No. 10, 2003, pp. 22172220. doi:10.1016/S0040-4039(03)00037-6

[72] S. R. S. S. Kotti, X. Xu, G. Li and A. D. Headly, "Efficient Nucleophilic Substitution Reactions of Highly Functionalized Allyl Halides in Ionic Liquid Media," Tetrahedron Letters, Vol. 45, No. 7, 2004, pp. 1427-1431. doi:10.1016/j.tetlet.2003.12.051

[73] S. T. Handy, "Grignard Reactions in Imidazolium Ionic Liquids,” The Journal of Organic Chemistry, Vol. 71, No. 12, 2006, pp. 4659-4662. doi:10.1021/jo060536o

[74] M. C. Law, K. Wong and T. H. Chen, "Grignard Reagents in Ionic Liquids," Chemical Communications, No. 23, 2006, pp. 2457-2459. doi:10.1039/b602718k

[75] T. Yoshino, S. Imori and H. Togo, "Efficient Esterification of Carboxylic Acids and Phosphonic Acids with Trialkyl Orthoacetate in Ionic Liquid,” Tetrahedron, Vol. 62, No. 6, 2006, pp. 1309-1317. doi:10.1016/j.tet.2005.09.147

[76] S. Imori and H. Togo, "Efficient Demethylation of N,NDimethylanilines with Phenyl Chloroformate in Ionic Liquids,” Synlett, No. 16, 2006, pp. 2629-2632.

[77] Y. Tsuchiya, Y. Izumisawa and H. Togo, "3-exo-tet Cyclization of 2,2-Disubstituted 1,3-Dihalopropanes with Indium in Aqueous and Ionic Liquid Solvent System,” Tetrahedron, Vol. 65, No. 36, 2009, pp. 7533-7537. doi:10.1016/j.tet.2009.06.123

[78] H. M. Meshram, P. N. Reddy, P. Vishnu, K. Sadashiv and J. S. Yadav, "A Green Approach for Efficient $\alpha$-Halogenation of $\beta$-Dicarbonyl Compounds and Cyclic Ketones Using $N$-Halosuccinimides in Ionic Liquids,” Tetrahedron Letters, Vol. 47, No. 6, 2006, pp. 991-995. doi:10.1016/j.tetlet.2005.11.141

[79] R. Rajagopal, D. V. Jarikote, R. J. Lahoti, T. Daniel and K. V. Srinivasan, "Ionic liquid Promoted Regioselective Monobromination of Aromatic Substrates with N-Bromosuccinimide," Tetrahedron Letters, Vol. 44, No. 9, 2003, pp. 1815-1817. doi:10.1016/S0040-4039(03)00092-3

[80] S. R. K. Pingali, M. Madhav and B. S. Jursic, “An Efficient Regioselective NBS Aromatic Bromination in the Presence of an Ionic Liquid," Tetrahedron Letters, Vol.
51, No. 10, 2010, pp. 1383-1385.

doi:10.1016/j.tetlet.2010.01.002

[81] P. Ruggli and M Herzog, “Über die Addition von Benzol an Symm. Dibrom-Diacetyl (Carbonylgruppen und Arom. Kohlenwasserstoffe, 1. Mitt.)," Helvetica Chimica Acta, Vol. 29, No. 1, 1946, pp. 95-101. doi:10.1002/hlca.19460290115

[82] G. L. Song, "Novel Disubstituted Phenylene-Linked Bisimidazole Derivatives: Facile Synthesis and Optical Properties," Helvetica Chimica Acta, Vol. 93, No. 12, 2010, pp. 2397-2405. doi:10.1002/hlca.201000014

[83] S. Kumar, V. Gawandi, N. Capito and R. Philips, "Substituent Effects on the Reaction of Beta-Benzoylalanines with Pseudomonas Fluorescens Kynureninase," Biochemistry, Vol. 49, No. 36, 2010, pp. 7913-7919.

[84] J. Wegmann and H. Dahn, "Über die Synthese von Aryl-ketolen durch Addition von Aromatischen Kohlenwasserstoffen in der Diacetylreihe (Carbonylgruppen und arom. Kohlenwasserstoffe, 2. Mitt.)," Helvetica Chimica Acta, Vol. 29, No. 1, 1949, pp. 101-103. doi:10.1002/hlca.19460290116

[85] A. Arrieta, I. Ganboa and C. Palomo, "Reagents and Synthetic Methods 38. 4-(Dimethylamino)pyridinium Bromide Perbromide as a New Brominating Agent for Organic Compounds," Synthetic Communications, Vol. 14, No. 10, 1984, pp. 939-945. doi:10.1080/00397918408063764

[86] P. Hou, "Synthesis of 2-Phenylthiazoles from AlphaTosyloxyketones and Thiobenzamide in $[\mathrm{Bmim}]\left[\mathrm{PF}_{6}\right]$ Ionic Liquid at Ambient Temperature," Journal of the Chinese Chemical Society, Vol. 53, Part 4, 2006, pp. 863866.

[87] J. Hummerle, M. Spina, M. Schnuerch and M. Mihovilovic, Synthesis 2008, 19, 3099-3107.

[88] M. Ueno and H. Togo, Synthesis 2004, 16, 2073-2077.

[89] F. Asinger, "Einwirkung von Schwefel und Ammoniak auf Propiophenon, $n$-Butyrophenon und $i$-Butyrophenon," Organic Chemistry and Biochemistry, Vol. 97, No. 5, 1966, pp. 1510-1522. doi:10.1007/BF00902605

[90] J. Hummerle, M. Schnuerch and M. D. Mihovilovic, “A Guideline for the Arylation of Positions 4 and 5 of Thiazole via Pd-Catalyzed Cross-Coupling Reactions," Tetrahedron, Vol. 65, No. 5, 2010, pp. 8051-8059. doi:10.1016/j.tet.2010.07.081

[91] Y. Ishiwata and H. Togo, “Title of This Paper,” Synlett, No. 17, 2008, pp. 2637-2641.

[92] J. N. Kar, R. C. Acharya and M. K. Rout, "Quaternization of Thiazoles,” The Journal of Organic Chemistry, Vol. 38, No. 12, 1973, pp. 2164-2166. doi:10.1021/jo00952a011 